\title{
Chest Computed Tomography Findings in COVID-19 Pneumonia from Tehran, Iran
}

\author{
Mohammad Javanbakht ${ }^{1}$, Ramezan Jafari ${ }^{2 \star}$, Mehdi Mesri ${ }^{3}$, Morteza Izadi ${ }^{4}$, Seyed Hassan Saadat ${ }^{1,5}$ \\ ${ }^{1}$ Nephrology and Urology Research Center, Baqiyatallah University of Medical Sciences, Tehran, Iran; ${ }^{2}$ Department of Radiology \\ and Health Research Center, Baqiyatallah University of Medical Sciences, Tehran, Iran; ${ }^{3}$ Quran and Hadith Research Center, \\ Baqiyatallah University of Medical Sciences, Tehran, Iran; ${ }^{4}$ Health Research Center, Baqiyatallah University of Medical Sciences, \\ Tehran, Iran; ${ }^{5}$ Behavioral Science Research Center, Lifestyle Institute, Baqiyatallah University of Medical Sciences, Tehran, Iran
}

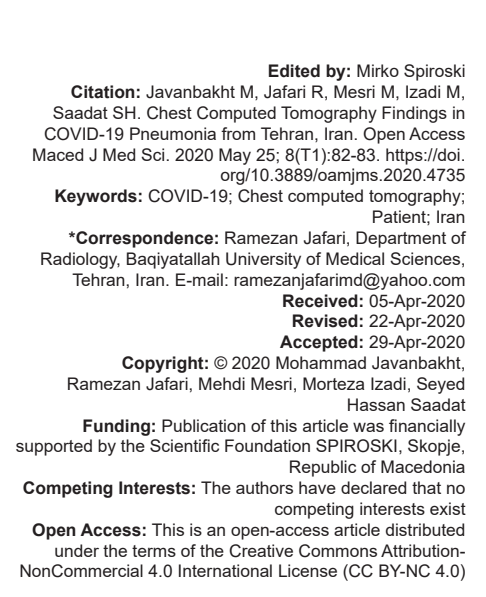

\section{Introduction}

Unfortunately, as is known, coronavirus disease 19 (COVID-19) with high transmission, extensive concern and challenge in public health the global, is spreading quickly [1], [2]. In parallel, the current diagnostic techniques for recognizing the virus have a great significance in the primary stage [2]. Nevertheless, computed tomography (CT) investigation widely applied in prognostication, monitoring the advance of disease, clinical management, and appraising the therapeutic strategies of COVID-19 pneumonia in worldwide cases [3], [4], [5].

\section{Findings}

In our report, CT images were obtained from 70 patients admitted to a Tehran Hospital after symptom onset between February 24, 2020, and April 2, 2020, who had confirmed COVID-19 pneumonia with age range $23-75$ years.
In our patients, the typical pattern of CT imaging features of COVID-19 patients comprised predominantly of multifocal subpleural patchy ground-glass opacity (GGO) in 64 cases (bilateral in 28 cases, unilateral in 12 cases, synchronous with patchy consolidation in 18 cases, and patchy GGO with crazy-paving pattern in 6 cases), only multifocal patchy consolidation and alveolar opacities in six cases, dense linear opacities in 16 case, and reverse halo sign in 22 cases (Figure 1). These lesions quickly evolved to become lung bilateral, multifocal, and diffuse with multilobar distribution in most patients (Figure 1).

On the other hand, in our case series, chest CT findings such as pulmonary nodules, masses, calcifications, tree-in-bud appearance, cystic changes, bronchiectasis, cavitation, pleural effusion, and mediastinal lymphadenopathy were not found.

\section{Conclusion}

In our case series, chest CT findings such as pulmonary nodules, masses, calcifications, tree-in-bud 

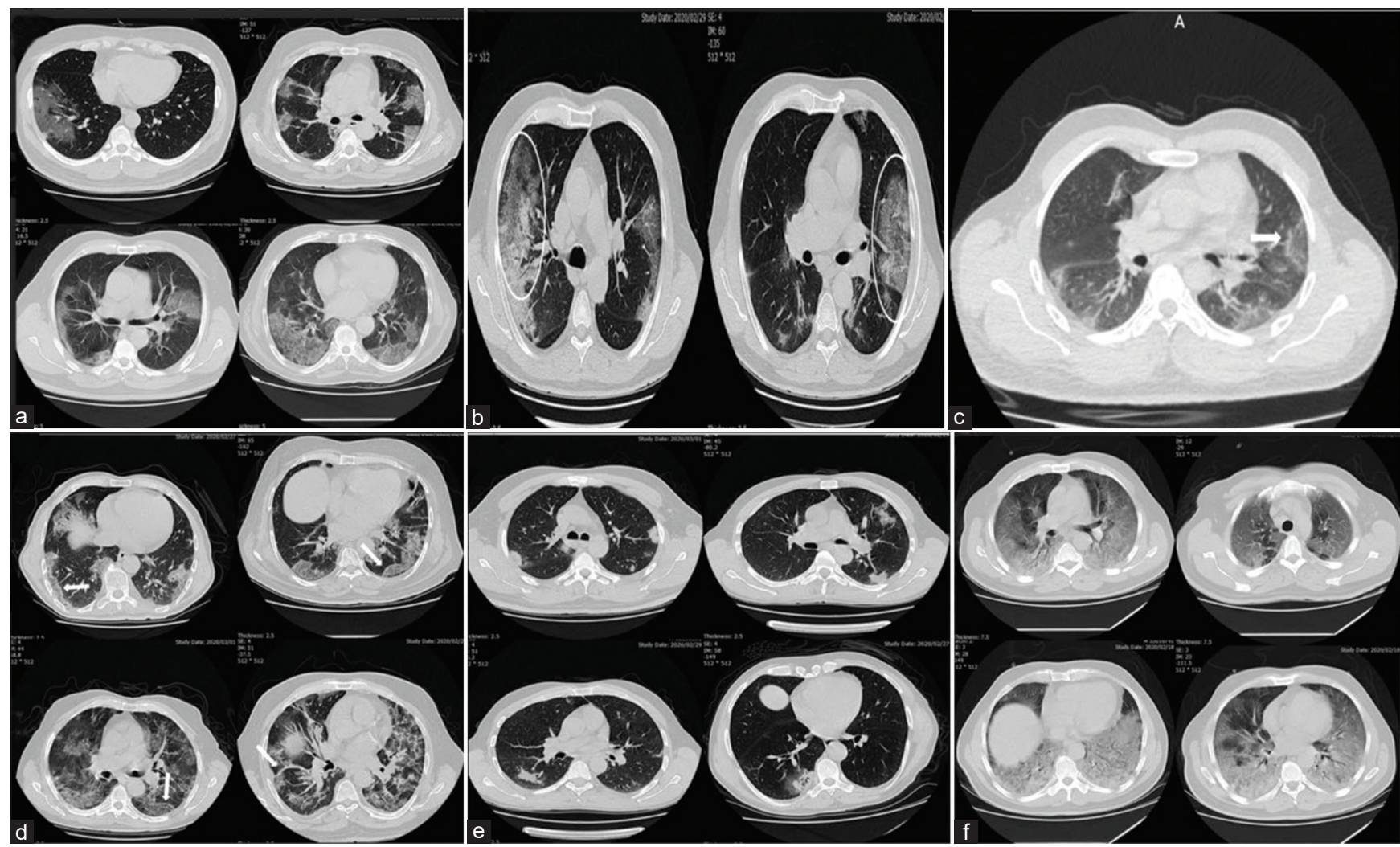

Figure 1: A) Multifocal subpleural patchy ground glass opacities with multilobar distribution on both lungs in multiple patients with confirmed COVID-19 infection. B) Mulifocal subpleural patchy ground glass opacities superimposed with interlobular and intralobular septal thickening compatible with crazy-paving pattern in a 61 year old male with COVID-19 infection. C) Reversed halo sign (white arrows) is a highly suggestive imaging finding for COVID-19 infection. D) Relatively dense linear opacities at boundary of ground glass opacities (white arrows) or in lung parenchyma is a common imaging finding at late stage of COVID-19 infection. E) Multifocal sub pleural patchy alveolar consolidation with multilobar distribution on both lungs in 57 year old male, which was a known case of chronic renal failure and positive PCR test for COVID-19 infection. F) Background of widespread ground glass opacities with an anteroposterior density gradient on both lung fields and consolidation in the most dependent areas compatible with ARDS in a 55 year old male with severe COVID-19 infection

appearance, cystic changes, bronchiectasis, cavitation, pleural effusion, and mediastinal lymphadenopathy were not found.

\section{Consent for publication}

Patients gave written informed consent for publication

\section{References}

1. Shereen MA, Khan SS, Kazmi A ,Bashir N, Siddique R. COVID-19 infection: Origin, transmission, and characteristics of human coronaviruses. J Adv Res. 2020;24:91-8. https://doi. org/10.1016/j.jare.2020.03.005

2. Bai HX, Hsieh B, Xiong Z, Halsey K, Whae CJ, Linh TT, et al. Performance of radiologists in differentiating COVID-19 from viral pneumonia on chest CT. Radiology 2020;2020;200823. https://doi.org/10.1148/radiol.2020200823

3. Chua F, Armstrong-James D, Desai SR, Barnett J, Kouranos V, Kon OM, et al. The role of CT in case ascertainment and management of COVID-19 pneumonia in the UK: Insights from high-incidence regions. Lancet Respir Med. 2020;8(5):P438-40.

4. Wang YX, Liu WH, Yang M, Chen W. The role of CT for Covid-19 patient's management remains poorly defined. Ann Transl Med. 2020;8(4):145. https://doi.org/10.21037/atm.2020.02.71

5. Ye Z, Zhang $Y$, Wang $Y$, Huang $Z$, Song B. Chest CT manifestations of new coronavirus disease 2019 (COVID19): A pictorial review. Eur Radiol. 2020;2020:1-9. https://doi. org/10.1007/s00330-020-06801-0

PMid:32193638 